Portland State University

PDXScholar

Mechanical and Materials Engineering Faculty

Publications and Presentations

\title{
A Mean Curvature Model for Capillary Flows in Asymmetric Containers and Conduits
}

Yongkang Chen

Portland State University

Noël Tavan

Portland State University

Mark M. Weislogel

Portland State University, weisloge@pdx.edu

Follow this and additional works at: https://pdxscholar.library.pdx.edu/mengin_fac

Part of the Fluid Dynamics Commons, and the Materials Science and Engineering Commons Let us know how access to this document benefits you.

\section{Citation Details}

Chen, Y., Tavan, N., \& Weislogel, M. M. (2012). A mean curvature model for capillary flows in asymmetric containers and conduits. Physics Of Fluids, 24(8), 082111. doi:10.1063/1.4749816

This Article is brought to you for free and open access. It has been accepted for inclusion in Mechanical and Materials Engineering Faculty Publications and Presentations by an authorized administrator of PDXScholar. Please contact us if we can make this document more accessible: pdxscholar@pdx.edu. 


\title{
A mean curvature model for capillary flows in asymmetric containers and conduits
}

\author{
Yongkang Chen, Noël Tavan, and Mark M. Weislogela) \\ Mechanical and Materials Engineering Department, Portland State University, Portland, \\ Oregon 97207-0751, USA
}

(Received 7 February 2012; accepted 1 August 2012; published online 31 August 2012)

\begin{abstract}
Capillarity-driven flows resulting from critical geometric wetting criterion are observed to yield significant shifts of the bulk fluid from one side of the container to the other during "zero gravity" experiments. For wetting fluids, such bulk shift flows consist of advancing and receding menisci sometimes separated by secondary capillary flows such as rivulet-like flows along gaps. Here we study the mean curvature of an advancing meniscus in hopes of approximating a critical boundary condition for fluid dynamics solutions. It is found that the bulk shift flows behave as if the bulk menisci are either "connected" or "disconnected." For the connected case, an analytic method is developed to calculate the mean curvature of the advancing meniscus in an asymptotic sense. In contrast, for the disconnected case the method to calculate the mean curvature of the advancing and receding menisci uses a well-established procedure. Both disconnected and connected bulk shifts can occur as the first tier flow of more complex compound capillary flows. Preliminary comparisons between the analytic method and the results of drop tower experiments are encouraging. (C) 2012 American Institute of Physics. [http://dx.doi.org/10.1063/1.4749816]
\end{abstract}

\section{INTRODUCTION}

When the forces of gravity are small compared to those of surface tension, system wettability and container shape can play a dominant role in fluid interfacial behavior. With control of such, one is able to guide numerous fluid transport outcomes precisely, passively, and without moving parts. Specifically, the unique dependence of capillarity-driven flows on container geometry has been a fruitful subject of study as it often concerns the system critical function of transporting fluids in macro-scale systems onboard spacecraft or in micro-scale fluidic devices on earth. Typical examples include flows along interior corners ${ }^{1-3}$ of polygonal containers and vane-wall gaps in circular cylinders. ${ }^{4}$ Consisting of single or multiple advancing menisci of the same hierarchic level and normally one receding meniscus, such flows are often considered elementary as compared to compound capillary flows such as those recently studied by Bolleddula $e t$ al. ${ }^{5}$

A "compound capillary flow" consists of capillarity-driven flows at different hierarchic levels over both local and global length scales with widely varying time scales. A typifying example of a large length-scale compound capillary flow was recently observed during the Capillary Flow Experiment (CFE) Vane-Gap experiments performed on the International Space Station ${ }^{6}$ (ISS). The Vane-Gap container geometry consists of a planar vane that pivots about the center of a right elliptical cylindrical container. The container and section dimensions are identified in Fig. 1 for the $127 \mathrm{~mm}$ tall container partially filled with perfectly wetting $10 \mathrm{cs}$ Polydimethylsiloxane (PDMS, silicone oil). The vane is not symmetric but forms two unequal gaps with the container wall as identified by $\delta_{1}<\delta_{2}$ in Fig. 1(c). Following simple discreet clockwise rotations of the vane, it was observed as expected that at specific vane angles, capillary flows, akin to corner flows, arise in the two gap regions forming slender rivulets. However, it was unexpectedly observed that at other

\footnotetext{
a)mmw@mme.pdx.edu.
} 


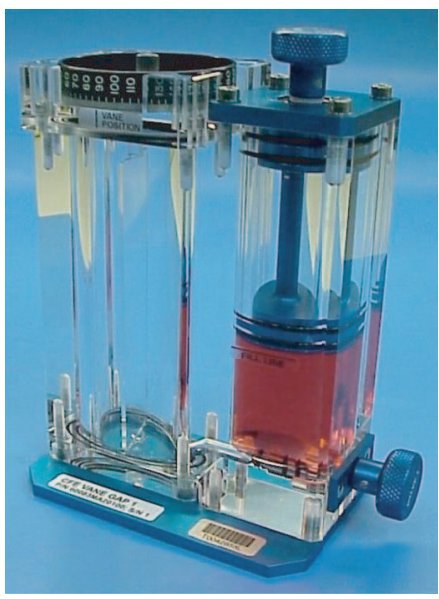

(a)

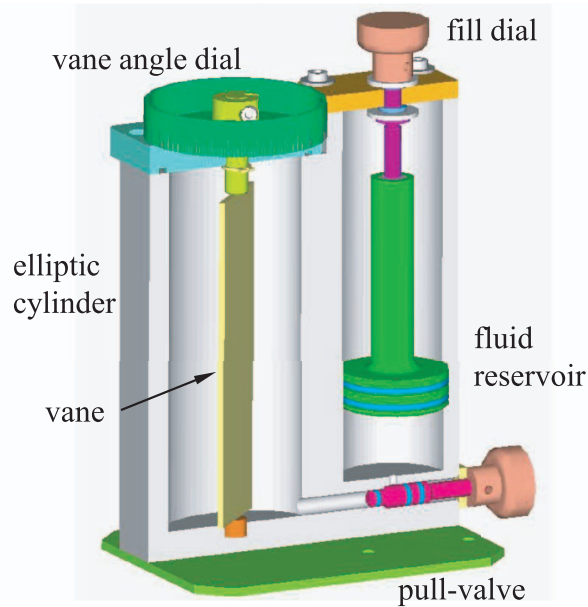

(b)

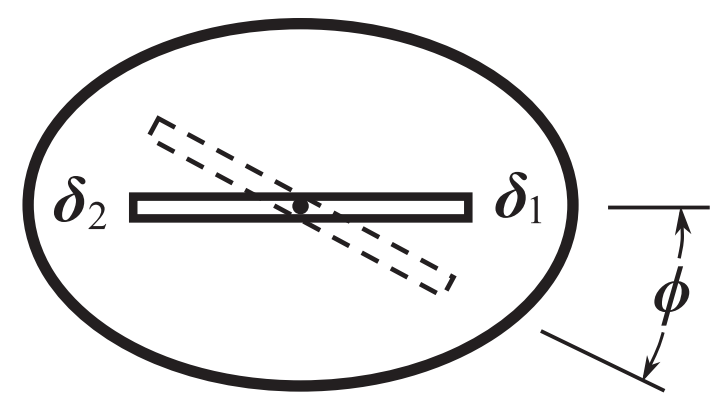

(c)

FIG. 1. (a) Image of CFE Vane-Gap vessel \#1 tested on International Space Station. (b) Solid model of vessel with key components identified. (c) Cross section of vane in right elliptical section: the major and minor axes of the ellipse are $50.800 \mathrm{~mm}$ and $33.866 \mathrm{~mm}$, respectively. The $127 \mathrm{~mm}$ tall elliptic cylinder is $30 \%$ filled with a perfectly wetting fluid $(10 \mathrm{cs}$ PDMS). The $114.300 \mathrm{~mm}$ tall vane section dimensions are $31.343 \mathrm{~mm}$ by $2.007 \mathrm{~mm}$. The vane is offset slightly from ellipse axis center of rotation such that at vane angle $\phi=90^{\circ}, \delta_{1}=\delta_{2} / 2=0.838 \mathrm{~mm}$.

vanes angles the entire fluid contents would shift from one side of the vane to the other as shown in Fig. 2. The "bulk shift" shown in Figs. 2(d) and 2(e) is referred to as a disconnected bulk shift (DBS) because the bulk menisci are separated by rivulet-like flows along the gaps. The dynamics of the process is considered as a compound capillary flow since the capillarity-driven bulk shift supplies the capillarity-driven gap flows. As a result, the bulk shift flow is viewed as a first tier flow arising from minuscule differences in bulk menisci curvature while the flows along the gap regions are considered as second tier flows. In Fig. 2(f), at a vane angle of $\phi=315^{\circ}$, a pure bulk shift takes place without the gap flows. This situation will be referred to as a connected bulk shift (CBS) and it is not a compound capillary flow.

During the ISS experiments it was observed that the flow in the small gap region precedes the flow in the large gap region which precedes that of the bulk shift flow as the vane is rotated clockwise from the position $\phi=0^{\circ}$ (refer Figs. 2(b)-2(d)). Each flow may be studied individually or simultaneously depending on the vane angle $\phi$. For example, if the vane is turned, from $\phi=0^{\circ}$ to $\phi=60^{\circ}$ as shown in Fig. 2(d), a compound capillary flow results where the small and large gap flows and the bulk shift flow occur simultaneously, albeit at different rates. Such flows are readily observed at microscales in terrestrial experiments, or in short duration drop tower experiments, but the long duration low-g environment of the ISS allows the unique systematic investigation of critical points where velocities approach zero and flow times are excessive ( $>30 \mathrm{~min})$. 


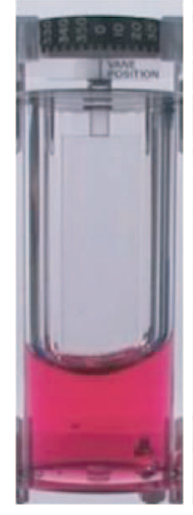

(a) $\phi=0^{\circ}$

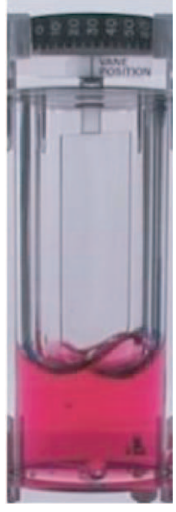

(b) $30^{\circ}$

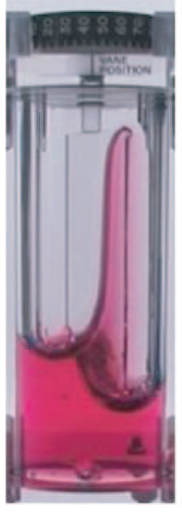

(c) $45^{\circ}$

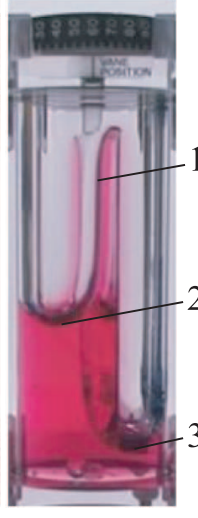

(d) $60^{\circ}$

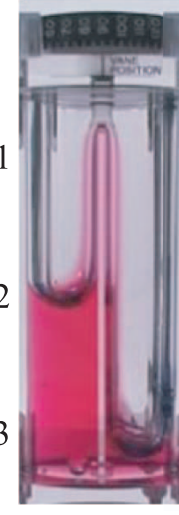

(e) $90^{\circ}$

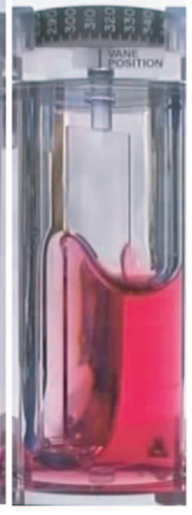

(f) $315^{\circ}$

FIG. 2. Sequence of equilibrium interface configurations during the Capillary Flow Experiment (CFE) Vane-Gap test with the vane rotated clockwise from position (a) $\phi=0^{\circ}$ to (f) $\phi=315^{\circ}$. In (d) $\phi=60^{\circ}$ advancing rivulet (\#1) gap flow, bulk advancing meniscus (\#2), and receding meniscus (\#3) are shown. See Fig. 1 for further details.

Because the amount of fluid involved in the bulk shift flow is significant, the mechanism for the shift must be understood to either avoid or exploit the phenomena depending on the application. It will be shown that in certain cases the bulk shift flow takes place due to slight geometric asymmetries at or even below container tolerance levels and that special measures should be taken to ensure that the desired effect is produced.

The focus of this study is not to explain why different scenarios arise when the vane is rotated to different positions in the CFE Vane-Gap experiments as these are reported separately. Instead, the goal of this work is to determine the mean curvature of the advancing bulk menisci since this quantity is essential to analyzing the disconnected (compound capillary) bulk shift or the connected (pure) bulk shift flows. Researchers de Lazzer et al. ${ }^{7}$ developed a closed form analytic approach to determine the mean curvature of a semi-infinite equilibrium meniscus which, approximated as quasi-steady, may be employed as an essential boundary condition for the analysis of numerous capillary flows. ${ }^{1,2,8}$ The present work is inspired by and extends that of de Lazzer et al. In what follows, the analytic method is developed which is then followed by its application to two model geometries. Comparisons with approximate solutions and experimental results are provided as well.

\section{ANALYTIC FORMULATION}

Knowledge of the mean curvature is foundational to capillary flows because it provides the motive pressure boundary condition for the flow. Researchers de Lazzer et al. ${ }^{7}$ explicitly derive an equation to solve for asymptotic equilibrium interface mean curvature in semi-infinite right cylindrical containers. Finn and $\mathrm{Neel}^{9}$ provide a general mathematical formulation and algorithm via the study of so-called C-singular solutions. These are equilibrium interface investigations, but the methods have been shown to be applicable to simple and compound quasi-steady flows with advancing and/or receding bulk menisci. ${ }^{1,8,10,11}$ In general, attention has been paid to flows where the curvatures of the advancing menisci are distinctly larger than those of the bulk receding menisci. Such flows usually mask the presence of second tier compound capillary flows. ${ }^{12}$ When the curvature of the advancing and receding bulk menisci are comparable, subtle changes in geometry amplify a host of compound capillary phenomena. For such flows it is imperative to identify the mean curvature of the bulk menisci which serve as the pressure boundary condition for both first and second tier capillary flows. A method is developed here to identify the mean curvature of the advancing bulk meniscus in cases where it is directly connected to the receding meniscus. It will be shown that in cases where the advancing bulk meniscus is disconnected from the receding bulk meniscus by second tier flows, the method developed by de Lazzer et al. is readily applied to determine the mean 


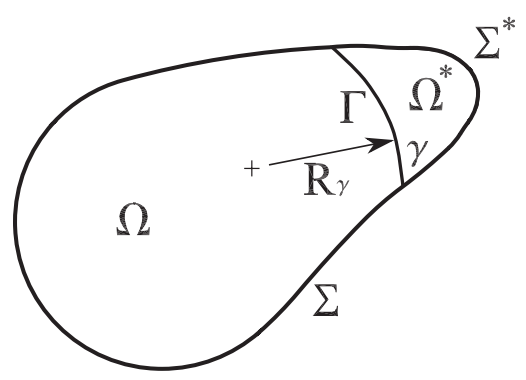

FIG. 3. A generic cross section of a right cylindrical container with $\operatorname{arc} \Gamma . \Omega$ and $\Sigma$ represent the entire domain and its boundary, respectively, whereas $\Omega^{*}$ represents a subdomain enclosed by boundary section $\Sigma^{*}$, and the $\operatorname{arc} \Gamma$, which meets the boundary $\Sigma$ at contact angle $\gamma$.

curvature. A simplified version of the theory is presented herein which is expected to be generalized further in a subsequent publication.

The mathematical approach taken here is known as Concus-Finn analysis. ${ }^{13-15}$ Following the tradition of that analysis, symbols with Greek letters are used to both denote an entity and its measure. For example, $\Omega$ denotes a problem domain and its area, $\Gamma$ an arc and its length, and $\Sigma$ the boundary and its length - the perimeter of the problem domain $\Omega$. The analysis begins with the Young-Laplace-Gauss equation which describes a constant mean curvature capillary surface of height $u(x, y)$ in a right cylindrical container on domain $\Omega$ with boundary $\Sigma$ as sketched in Fig. 3, thus

$$
\operatorname{div} \mathbf{T} u=2 H,
$$

where

$$
\mathbf{T} u \equiv \frac{\nabla u}{\sqrt{1+|\nabla u|^{2}}},
$$

and $H$ is the mean curvature of the surface. The boundary condition is

$$
v \cdot \mathbf{T} u=\cos \gamma,
$$

where $v$ is the exterior unit normal on $\Sigma$ and $\gamma$ is the contact angle at which the fluid meets the cylinder wall.

In Concus-Finn analysis a subdomain $\Omega^{*}$ enclosed by a section of the boundary $\Sigma^{*}$ and $\operatorname{arcs} \Gamma$ are used to identify the existence of an equilibrium capillary surface over the entire domain $\Omega$ as sketched in Fig. 3. Note that $\Omega^{*}$ is on the convex side of the $\operatorname{arc} \Gamma$ and the curvature of $\Gamma$ is given by

$$
2 H=\frac{\Sigma \cos \gamma}{\Omega} .
$$

A functional

$$
\Phi=\Gamma-\Sigma^{*} \cos \gamma+2 H \Omega^{*}
$$

is evaluated over every $\Omega^{*}$. In general, it can be shown ${ }^{16}$ that equilibrium capillary surfaces fail to exist over $\Omega$ for the given boundary condition on $\Sigma$ when $\Phi \leq 0$ for an $\Omega^{*}$ which is neither the null set nor all of $\Omega$. However, according to Finn and $\mathrm{Neel}^{9}$ there exist C-singular solutions that may be represented by $\operatorname{arcs} \Gamma_{\mathrm{o}}$ inside of $\Omega$, an example sketch of which is provided in Fig. 4(a). For clarity, the geometry is chosen such that only one C-singular solution is present. $\Gamma_{\mathrm{o}}$ of Fig. 4(a) is the same as $\Gamma$ shown in Fig. 3 only when $\Phi=0$. When $\Phi<0, \Gamma_{\mathrm{o}}$ must be found using an alternative approach. In Fig. 4(a) $\Gamma_{\mathrm{o}}$ and a section of the boundary $\Sigma_{\mathrm{o}}$ enclose a subdomain $\Omega_{\mathrm{o}}$ on the concave side of $\Gamma_{\mathrm{o}}$. The complementary part of $\Omega_{\mathrm{o}}$ on the convex side of $\Gamma_{\mathrm{o}}$ is represented by $\bar{\Omega}_{\mathrm{o}}$ and $\Omega=\Omega_{\mathrm{o}}+\bar{\Omega}_{\mathrm{o}}$, and the complementary part of $\Sigma_{\mathrm{o}}$ is $\bar{\Sigma}_{\mathrm{o}}$ and $\Sigma=\Sigma_{\mathrm{o}}+\bar{\Sigma}_{\mathrm{o}}$.

As shown in Fig. 4(a), for a C-singular solution, from the concave side of $\Gamma_{\mathrm{o}}$ the surface asymptotically approaches a cylindrical surface as $l \rightarrow \infty$ which is an extrusion of $\Gamma_{0}$, where $l=l(t)$ is a measure of the vertical distance between bulk menisci. The portion of the surface on $\bar{\Omega}_{0}$ rises to infinity since a finite-height single-valued surface does not exist on this subdomain. Imagine that in a 


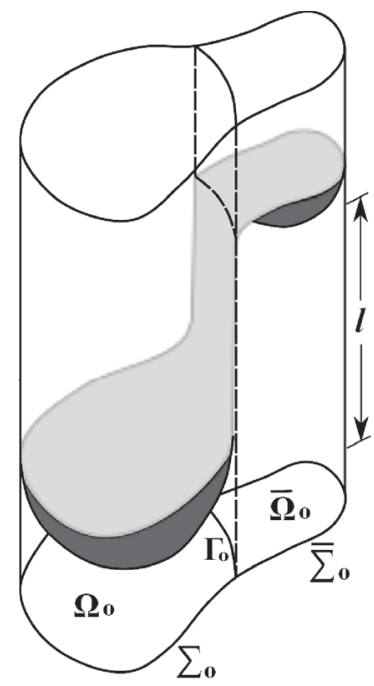

(a)

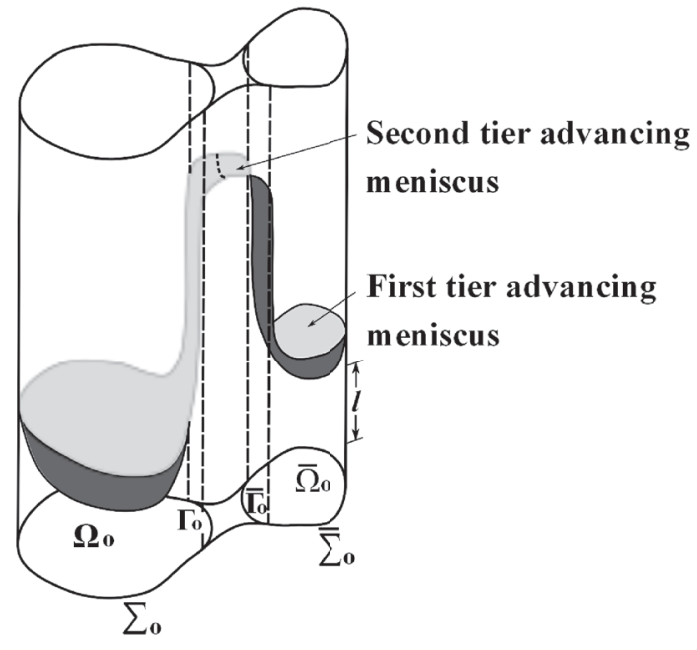

(b)

FIG. 4. (a) "Connected" bulk menisci: C-singular solution surface in a cylindrical container of domain $\Omega=\Omega_{\mathrm{o}}+\bar{\Omega}_{\mathrm{o}}$. An extrusion of the $\operatorname{arc} \Gamma_{\mathrm{o}}$ is shown as the vertical cylindrical surface which the free surface on $\Omega_{\mathrm{o}}$ approaches asymptotically. (b) "Disconnected" bulk menisci separated by a second tier capillary structure in the form of an advancing rivulet ahead of the advancing bulk meniscus. From a dynamics perspective, the motion of such configurations is considered as compound capillary flows. $l$ is a characteristic elevation difference between bulk menisci.

terrestrial gravity situation the interface in a cylindrical container such as the one shown in Fig. 4(a) possesses a certain equilibrium configuration. When the gravity is turned off, given that the generalized Concus-Finn condition $\Phi \leq 0$ is satisfied, part of the surface on $\bar{\Omega}_{0}$ advances to form an advancing bulk meniscus while the part of the surface on $\Omega_{\mathrm{o}}$ forms a receding bulk meniscus. ${ }^{5}$ The advancing meniscus rises to infinity, i.e., $l=l(t) \rightarrow \infty$ to establish the $\mathrm{C}$-singular solution. However, before this ultimate condition, in an asymptotic sense the advancing bulk meniscus is likely to establish a quasisteady state with its own constant mean curvature. This claim becomes the enabling assumption of the current analysis which seeks to evaluate the mean curvature of the advancing meniscus.

To do this it is first necessary to determine the constant mean curvature $H_{\mathrm{o}}$ of the receding bulk meniscus. As shown by de Lazzer et al. ${ }^{7}$ and Finn and $\mathrm{Neel}^{9}$ this can be achieved by integrating Eq. (2.1) over the subdomain $\Omega_{\circ}$ which yields

$$
\iint_{\Omega_{\circ}} \operatorname{div} \mathbf{T} u \mathrm{~d} A=\iint_{\Omega_{\circ}} 2 H_{\circ} \mathrm{d} A .
$$

Applying the two-dimensional divergence theorem to Eq. (2.6) yields

$$
\Sigma_{\circ} \cos \gamma+\Gamma_{\circ}=2 H_{\circ} \Omega_{\circ} .
$$

The boundary conditions are contact angle $\gamma$ on the regular boundary $\Sigma_{\circ}$ and $\gamma=0$ on $\Gamma_{\circ}$. Rearranging (2.7) yields

$$
2 H_{\circ}=\frac{\Sigma_{\circ} \cos \gamma+\Gamma_{\circ}}{\Omega_{\circ}} .
$$

In most cases, it is not straightforward to find $H_{\circ}$ as both $\Omega_{\mathrm{o}}$ and $\Sigma_{\mathrm{o}}$ are dependent on the location of $\Gamma_{\circ}$ which is to be determined and a trial and error process is often pursued. An analytical solution only exists for certain geometries such as polygons with uniform contact angle as shown by de Lazzer et al. ${ }^{7}$ Weislogel and Collicott, ${ }^{10}$ and others.

Since $\gamma=0$ is the asymptotic condition of the receding bulk meniscus on $\Gamma_{\mathrm{o}}$, it must be the case that $\gamma=180^{\circ}$ is the asymptotic condition of the advancing bulk meniscus on $\Gamma_{\mathrm{o}}$. In a similar 
fashion to $H_{o}$, the constant mean curvature $\bar{H}_{\mathrm{o}}$ of the advancing meniscus can be written as

$$
2 \bar{H}_{\mathrm{o}}=\frac{\bar{\Sigma}_{\mathrm{o}} \cos \gamma-\Gamma_{\mathrm{o}}}{\bar{\Omega}_{\mathrm{o}}}
$$

and Eq. (2.5) can be rearranged to yield

$$
2 H=\frac{\Phi}{\Omega^{*}}+2 H^{*}
$$

where

$$
2 H^{*}=\frac{\Sigma^{*} \cos \gamma-\Gamma}{\Omega^{*}} .
$$

A direct relation between $\bar{H}_{\mathrm{o}}$ and $H_{\mathrm{o}}$ can be derived when the subdomains $\Omega_{\mathrm{o}}$ and $\bar{\Omega}_{\mathrm{o}}$ are complimentary, i.e., $\Omega_{0}+\bar{\Omega}_{0}=\Omega$. Substituting $\bar{\Sigma}_{\mathrm{o}}=\Sigma-\Sigma_{\mathrm{o}}$ into Eq. (2.9) and applying Eqs. (2.8) and (2.10) gives rise to

$$
2 \bar{H}_{\mathrm{o}}=\frac{2 H_{\mathrm{o}}}{\bar{\Omega}_{\mathrm{o}}}\left(\frac{2 H}{2 H_{\mathrm{o}}} \Omega-\Omega_{\mathrm{o}}\right)=\frac{2 H_{\mathrm{o}}}{\bar{\Omega}_{\mathrm{o}}}\left(\frac{\Omega}{2 H_{\mathrm{o}}}\left(\frac{\Phi}{\Omega^{*}}+2 H^{*}\right)-\Omega_{\mathrm{o}}\right) .
$$

When $\Phi=0, \Gamma$ is the same as $\Gamma_{\mathrm{o}}$ such that $H_{\mathrm{o}}=H=H^{*}$ and consequently, $\bar{H}_{\mathrm{o}}=H_{\mathrm{o}}$. This implies that at the critical Concus-Finn wetting condition, even though part of the interface extends to infinity, in an asymptotic sense the whole interface will have a constant mean curvature which is determined by the shape of the cross section of the container. In other words, the interface achieves equilibrium once the interface reaches infinity on $\bar{\Omega}_{\mathrm{o}}$. This is not so when $\Phi<0$, where $\bar{H}_{\mathrm{o}}>H_{\mathrm{o}}$ since $H>H_{\mathrm{o}}$. Equation (2.11) provides a quantitative measure of the relation between the quantity $\Phi$ and the mean curvature difference across the interface, although in an implicit form and for a simple configuration where there is only one $\mathrm{C}$-singular solution present.

A more general situation arises for compound capillary flows (for simplicity, only two levels of flow are considered here) when the first tier bulk advancing and receding menisci are separated by second tier capillary flows such that $\bar{\Sigma}_{\mathrm{o}}=\Sigma-\Sigma_{\mathrm{o}}$ may not be valid rendering Eq. (2.11) inapplicable. In such situations, both first and second tier flows are present and both advancing and receding bulk menisci can be bound by multiple arcs that represent $\mathrm{C}$-singular solutions. This situation is depicted in the sketch of Fig. 4(b) where an advancing rivulet divides the bulk menisci. The receding meniscus is always on the concave side of the arcs and Eq. (2.8) remains valid except that $\Gamma_{\mathrm{o}}$ is a summation of the length of all the arcs. The second tier advancing menisci (often rivulet like) are always on the convex side of all the arcs. The first tier advancing meniscus, however, can be on the concave side of the arcs connected to the second tier advancing meniscus where $\gamma=0$, while also on the convex side of the remainder of arcs connected to the receding meniscus where $\gamma=180^{\circ}$. Consequently, Eq. (2.9) should be generalized to

$$
2 \bar{H}_{\mathrm{o}}=\frac{\bar{\Sigma}_{\mathrm{o}} \cos \gamma+\bar{\Gamma}_{\mathrm{o}}-\Gamma_{\mathrm{o}}}{\bar{\Omega}_{\mathrm{o}}},
$$

with the advancing bulk meniscus on the concave side of $\bar{\Gamma}_{\mathrm{o}}$ while on the convex side of $\Gamma_{\mathrm{o}}$, see Fig. 12(b). If the advancing bulk meniscus is on the concave side of all the arcs then Eq. (2.12) degenerates to Eq. (2.8)

Theoretically, the mean curvature of the bulk advancing and receding menisci reaches $2 \bar{H}_{\mathrm{o}}$ and $2 H_{\mathrm{o}}$ asymptotically as $l \rightarrow \infty$. In practice, however, both $2 \bar{H}_{\mathrm{o}}$ and $2 H_{\mathrm{o}}$ can be used to approximate the mean curvature of the advancing and receding bulk menisci to aid, for example, the analysis of the fluid dynamics of the process. Two sample geometries are analyzed herein as models to demonstrate the procedure.

\section{MODEL GEOMETRIES}

To both guide and verify the analysis, drop tower tests are performed with test cells of two cross sectional shapes: asymmetric conjoined right circular cylinders and vane-partitioned right 


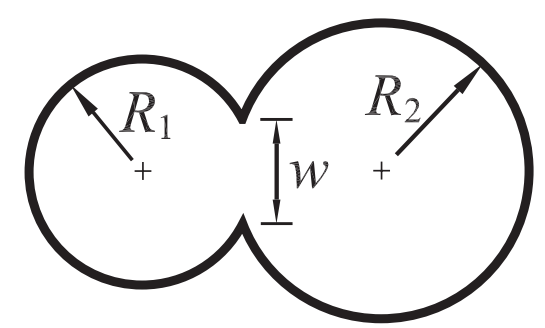

FIG. 5. Cross section of asymmetric conjoined circular cylinders.

rectangles. The experiments were conducted at the $2.2 \mathrm{~s}$ drop tower at NASA's Glenn Research Center. ${ }^{17}$ All tests are performed in the pre-wetted surface condition. ${ }^{18}$ Only a portion of the data will be presented here with further details reported by Tavan. ${ }^{19}$

\section{A. Asymmetric conjoined circular cylinders}

Asymmetric conjoined circular cylinders are designed for studying the dynamics of connected bulk menisci leading to pure bulk shift flow. A sketch of the cross section is provided in Fig. 5 . Assuming $\gamma=0$, the area and perimeter of the cross section may be determined by

$$
\begin{aligned}
\Omega= & \pi\left(R_{1}^{2}+R_{2}^{2}\right)-R_{1}^{2} \sin ^{-1} \frac{w}{2 R_{1}}+\frac{w R_{1}}{2} \cos \left(\sin ^{-1} \frac{w}{2 R_{1}}\right)-R_{2}^{2} \sin ^{-1} \frac{w}{2 R_{2}} \\
& +\frac{w R_{2}}{2} \cos \left(\sin ^{-1} \frac{w}{2 R_{2}}\right), \\
\Sigma= & 2 \pi\left(R_{1}+R_{2}\right)-2 R_{1} \sin ^{-1} \frac{w}{2 R_{1}}-2 R_{2} \sin ^{-1} \frac{w}{2 R_{2}},
\end{aligned}
$$

and the mean curvature of the $\operatorname{arc} \Gamma$ is

$$
\frac{1}{R_{\gamma}} \equiv 2 H=\frac{\Sigma \cos \gamma}{\Omega}
$$

It is tempting to assume that an equilibrium capillary surface fails to exist whenever there is asymmetry in the geometry such that $R_{1} \neq R_{2}$. It will be shown that this is not the case. There are at least two $\{\Gamma ; \gamma\}$ configurations for identifying the existence of the equilibrium surfaces as shown in Fig. 6. From Eq. (2.5), computations show that $\Phi$ becomes negative only for the configuration shown in Fig. 6(a), which is plotted in Fig. 7 and reveals that $\Phi$ changes sign at $\lambda \equiv R_{2} / R_{1} \approx 1.13499$. In other words, for $\gamma=0$, only values $\lambda \geq 1.13 \ldots$ result in bulk shifts.

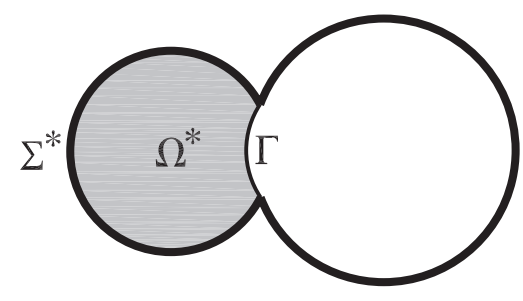

(a)

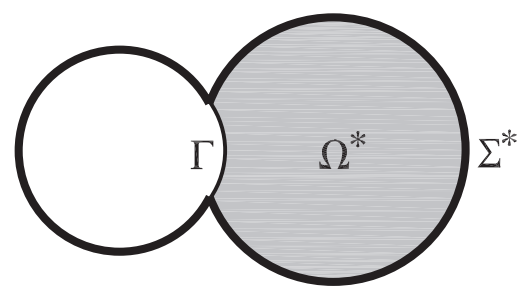

(b)

FIG. 6. $\{\Gamma ; \gamma\}$ configurations for asymmetric conjoined circular cylinders. 


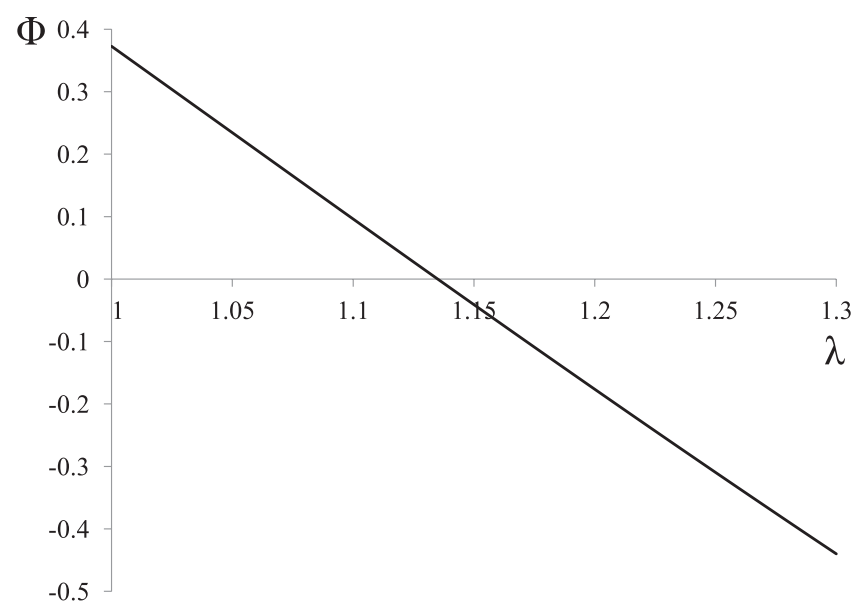

FIG. 7. $\Phi$ as a function of $\lambda=R_{2} / R_{1}$ from Eq. (2.5) for asymmetric conjoined circular cylinders. A linear fit yields $\Phi=-2.7159 \lambda+3.0849$.

Equilibrium surfaces fail to exist on $\Omega$ once $\Phi \leq 0$ and Eqs. (2.8) and (2.9) can be used to evaluate the mean curvature of the receding and advancing menisci. For the receding meniscus,

$$
\begin{gathered}
\Omega_{\mathrm{o}}=\pi R_{2}^{2}-R_{2}^{2} \sin ^{-1} \frac{w}{2 R_{2}}+\frac{w R_{2}}{2} \cos \left(\sin ^{-1} \frac{w}{2 R_{2}}\right)+R_{\mathrm{o}}^{2} \sin ^{-1} \frac{w}{2 R_{\mathrm{o}}}-\frac{w R_{\mathrm{o}}}{2} \cos \left(\sin ^{-1} \frac{w}{2 R_{\mathrm{o}}}\right), \\
\Sigma_{\mathrm{o}}=2 \pi R_{2}-2 R_{2} \sin ^{-1} \frac{w}{2 R_{2}},
\end{gathered}
$$

and

$$
\Gamma_{\mathrm{o}}=2 R_{\mathrm{o}} \sin ^{-1} \frac{w}{2 R_{\mathrm{o}}} .
$$

Knowing $R_{\mathrm{o}} \equiv 1 /\left(2 H_{\mathrm{o}}\right)$, Eqs. (3.1)-(3.3) can be substituted into Eq. (2.8) to solve for $R_{\mathrm{o}}$. Unfortunately, an analytical expression of $R_{\mathrm{o}}$ has not been found, and $R_{\mathrm{o}}$ must be solved numerically for each case.

A graphical demonstration of a quasi-steady surface in the bulk shift flow of the conjoined circular cylinders is shown in Fig. 13(a). Since the pure bulk shift flow is not a compound capillary flow it follows that $\bar{\Omega}_{\mathrm{o}}=\Omega-\Omega_{\mathrm{o}}$ and $\bar{\Sigma}_{\mathrm{o}}=\Sigma-\Sigma_{\mathrm{o}}$ as shown in Fig. 8, and that either Eq. (2.9) or (2.11) can be used to calculate $\bar{H}_{\mathrm{o}}$. The pure bulk shift flow in finite containers for this geometry has been studied both analytically and experimentally as briefly summarized by Bolleddula et al. ${ }^{5}$ In that study it is shown that the displacement of the advancing meniscus minimum $l$ is proportional to $t^{1 / 2}$ such that

$$
l \equiv m t^{1 / 2}
$$

where the coefficient $m$ is determined by many parameters including the mean curvatures of the menisci. The experimental flows are complicated by non-negligible fabrication tolerances, surface

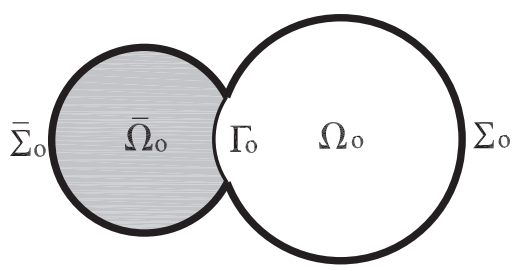

FIG. 8. Bulk shift in the asymmetric conjoined circular cylinders. 


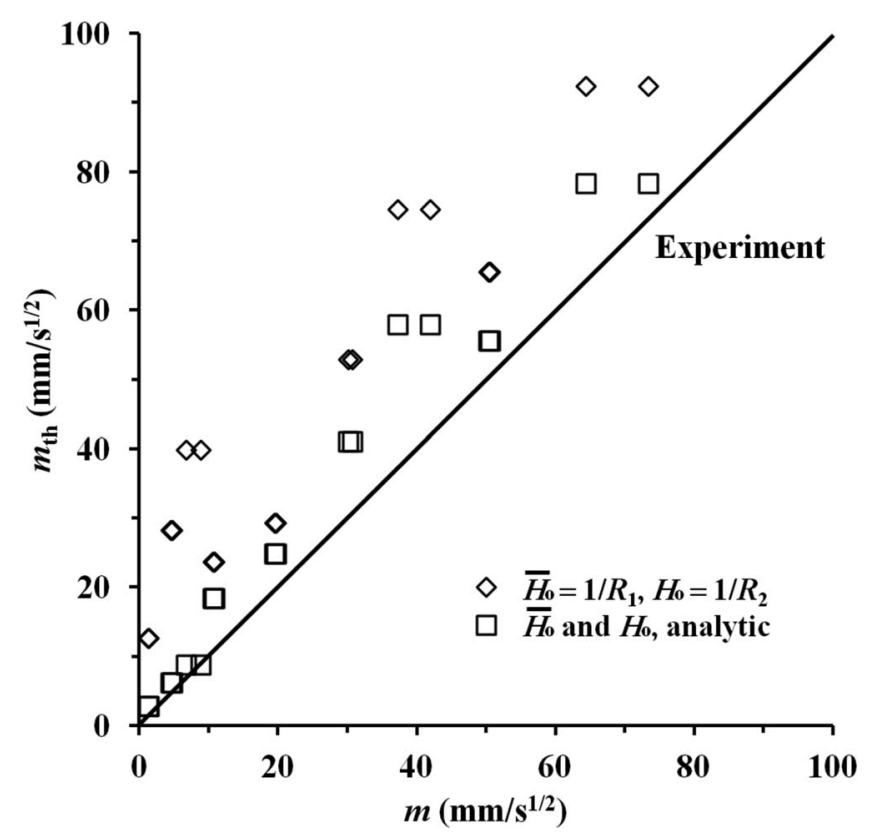

FIG. 9. Comparison of Tavan's ${ }^{19}$ coefficient $m$ from Eq. (3.4) for pure bulk shift capillary rise in drop tower tests of asymmetric conjoined circular cylinders: the experiments are predicted with $m_{\text {th }}$ values using meniscus mean curvature evaluated using both cylinder radii ${ }^{19}$ (diamonds) and the present method (squares).

conditions, and advancing non-uniform dynamic contact line and contact angle effects. As a start, Tavan ${ }^{19}$ estimates the mean curvatures of the menisci by the radii of the cylinders. In this study the mean curvatures of the menisci are calculated with the above formulations, i.e., Eq. (2.9), which are used in turn to calculate $m$, which is then compared to the experiments in Fig. 9. It appears that on average, using the formulations from this study to calculate the mean curvatures, the flow model overpredicts the data by $41 \%$ whereas using the radii of the cylinders the flow model overpredicts the data by $245 \%$.

\section{B. Vane-partitioned rectangles (VPR)}

A sketch of the VPR is shown in Fig. 10. The addition of the vane to an otherwise simply connected rectangular domain introduces substantial complexity. The complication arises when the dimension of the vane is significant enough such that C-singular solutions exist not only in the corner regions of the rectangle, ${ }^{20,21}$ but also the gap regions between the vane and the walls of the rectangle. ${ }^{22}$ It should be noted that the presence of the vane is not negligible even when its cross sectional area is zero as both sides of the vane are counted when calculating the perimeter of the entire problem domain as shown below.

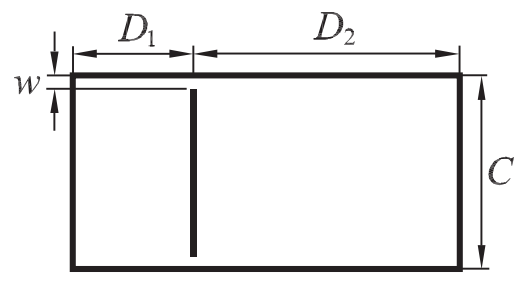

FIG. 10. Cross section of the vane-partitioned right rectangle. 


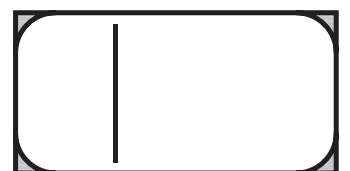

(a) VPRa

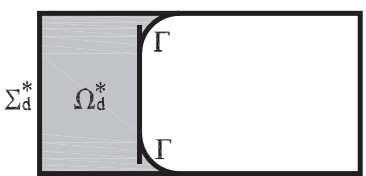

(d) VPRd

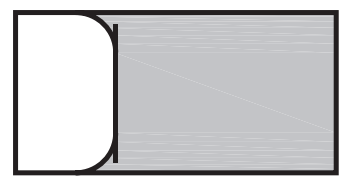

(f) VPRf

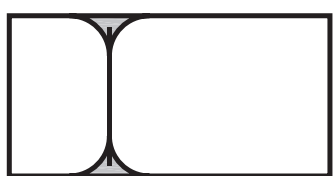

(b) VPRb

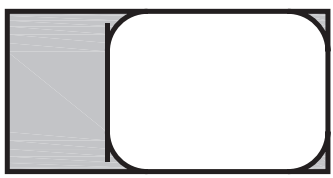

(e) VPRe

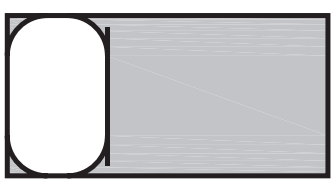

(g) VPRg

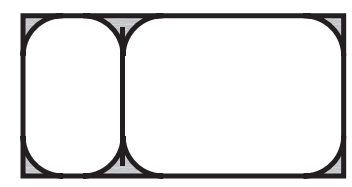

(c) VPRc

FIG. 11. $\{\Gamma ; \gamma\}$ configurations for the vane-partitioned rectangle domain. Only VPRa, VPRc, and VPRe are relevant to the present analysis.

Assuming $\gamma=0$ and a zero-thickness vane, the area and perimeter of the cross section may be written as

$$
\begin{gathered}
\Omega=\left(D_{1}+D_{2}\right) C, \\
\Sigma=2\left(D_{1}+D_{2}+2 C-2 w\right),
\end{gathered}
$$

and the mean curvature of the arc $\Gamma$ is

$$
\frac{1}{R_{\gamma}} \equiv 2 H=\frac{\Sigma \cos \gamma}{\Omega}=\frac{2\left(D_{1}+D_{2}+2 C-2 w\right)}{\left(D_{1}+D_{2}\right) C} .
$$

The complexity of the geometry manifests in the number of possible $\{\Gamma ; \gamma\}$ configurations for identifying the existence of the equilibrium surfaces. At least seven configurations may be identified as shown in Fig. 11. As arc $\Gamma$ represents $C$-singular solution in the case of $\Phi=0$, these configurations also represent the locations of potential C-singular solutions. Configuration VPRa shown in Fig. 11(a) includes four identical subdomains in each corner, while VPRb includes two identical subdomains. Each subdomain in VPRa, VPRb, or any combinations of VPRa and VPRb subdomains are legitimate configurations that are ignored because such configurations are merely subsets of VPRa, VPRb, or VPRc.

It is rather straightforward to evaluate $\Phi$ for each configuration. Only the formulations for VPRa are listed as an example here. These are written as

$$
\begin{gathered}
\Omega_{a}^{*}=(4-\pi) R_{\gamma}^{2}, \\
\Sigma_{a}^{*}=8 R_{\gamma}, \\
\Gamma_{a}=2 \pi R_{\gamma},
\end{gathered}
$$

and consequently

$$
\Phi_{a}=(\pi-4) R_{\gamma},
$$

where $\Omega_{a}^{*}$ is the summation of the four shaded corner areas and $\Sigma_{a}^{*}$ is the summation of the boundaries of the four shaded corner areas excluding the summation of the length of arcs $\Gamma$ included in $\Gamma_{a}$. It 
follows that $\Phi$ for the other configurations may be written as

$$
\begin{gathered}
\Phi_{b}=(\pi-4) R_{\gamma}+4 w, \\
\Phi_{c}=2(\pi-4) R_{\gamma}+4 w, \\
\Phi_{d}=\frac{1}{2}(\pi-4) R_{\gamma}-\left(2 D_{1}+2 C-4 w\right)+\frac{C D_{1}}{R_{\gamma}}, \\
\Phi_{e}=(\pi-4) R_{\gamma}-\left(2 D_{1}+2 C-4 w\right)+\frac{C D_{1}}{R_{\gamma}}, \\
\Phi_{f}=\frac{1}{2}(\pi-4) R_{\gamma}-\left(2 D_{2}+2 C-4 w\right)+\frac{C D_{2}}{R_{\gamma}},
\end{gathered}
$$

and

$$
\Phi_{g}=(\pi-4) R_{\gamma}-\left(2 D_{2}+2 C-4 w\right)+\frac{C D_{2}}{R_{\gamma}},
$$

where subscripts $b-g$ correspond to the configurations VPRb-VPRg shown in Fig. 11. A few key observations may be made $(\gamma=0)$ :

(1) $\Phi_{a}<0$, which implies that equilibrium surfaces always fail to exist and a C-singular solution always exists in each corner.

(2) $\Phi_{c}=\Phi_{a}+\Phi_{b}$, which means that with $\Phi_{a}<0$, if $\Phi_{b} \leq-\Phi_{a}$, then $\Phi_{c} \leq 0$. This implies that the C-singular solutions in VPRb, in the case of $\Phi_{b} \leq 0$, cannot exist independently and it must combine with the C-singular solutions in VPRa to form the C-singular solutions of VPRc. In fact, a critical value for the gap size $w$ can be found by substituting Eq. (3.5) into Eq. (3.6) and letting $\Phi_{c}=0$ which yields

$$
w_{\mathrm{cr}}=\frac{P-\sqrt{P^{2}+2(\pi-4)(P-2 C) C}}{4},
$$

where $P=D_{1}+D_{2}+2 C$. C-singular solutions depicted in VPRc exist when $w \leq w_{c r}$ for the given dimensions of the rectangle.

(3) Configurations VPRd-VPRg identify the C-singular solutions where bulk shifts are present. The C-singular solutions in configurations VPRd and VPRf cannot exist independently since the C-singular solutions in the two corners on the right side of the rectangle in VPRd and the left side of the rectangle in VPRf always exist. As a result either VPRe or VPRg must be evaluated to identify C-singular solutions producing a bulk shift.

(4) The configuration VPRa or VPRc overlaps with VPRe or VPRg. For cases where there are $\mathrm{C}$-singular solutions in VPRe or VPRg, a close examination reveals that the C-singular solutions in VPRa (when $\Phi_{b}>-\Phi_{a}$ ) or VPRc (when $\Phi_{b} \leq-\Phi_{a}$ ) should be superposed onto the C-singular solutions in VPRe or VPRg to form a global C-singular solution for the entire problem domain.

It can be shown that $\Phi_{c}=\Phi_{e}+\Phi_{g}$, and thus when $D_{1}=D_{2}, \Phi_{c}=2 \Phi_{e}=2 \Phi_{g}$. For the special case of $\Phi_{c}=0$ with $D_{1}=D_{2}, \Phi_{e}=\Phi_{g}=0$. It is certain that C-singular solutions exist in VPRc. The question to ask is what happens with VPRe and VPRg? For $D_{1}=D_{2}, \operatorname{VPRe}$ and VPRg are symmetric about the centerline of the vane. It is speculated that there is no bulk shift due to perfect symmetry even though $\Phi_{e}=\Phi_{g}=0$ for this special case. Symmetry is broken for an infinitesimal displacement of the vane to either side of the center of the rectangle since $\Phi_{c}=0$ still holds while $\Phi_{e}=-\Phi_{g}$. For cases when $D_{1}<D_{2}$, then $\Phi_{e}<0$, and $\Phi_{g}>0$. Consequently, the global C-singular solution is formed by superposing the C-singular solution of VPRc onto VPRe; the C-singular solutions in the two corners on the right side of the rectangle are common to both solutions. These solutions correspond to a first tier receding bulk meniscus flow supplying two second tier corner flows. Similarly, the C-singular solutions in the two corners on the left side of the rectangle correspond to a first tier advancing bulk meniscus supplying two second tier corner flows. 


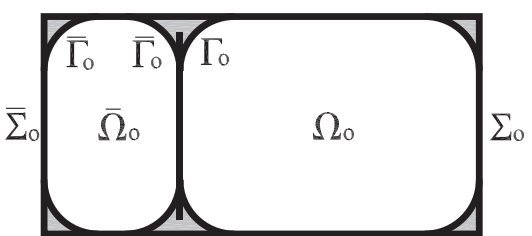

(a)

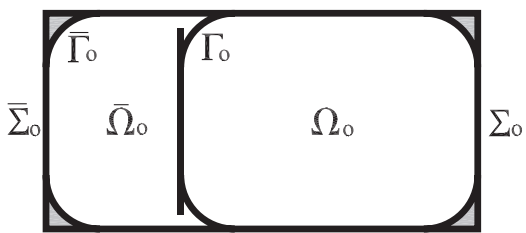

(b)

FIG. 12. Two scenarios concerning the bulk shift in the vane-partitioned rectangle. (a) Advancing and receding bulk menisci are separated (disconnected) by rivulet-shaped advancing menisci in the gap regions (DBS). (b) Connected advancing and receding bulk menisci (CBS).

Furthermore, the advancing bulk meniscus is separated from the receding bulk meniscus by rivulet-shaped advancing menisci in the gap regions as sketched in Fig. 12(a) with a quasi-steady surface shown in Fig. 13(b). This type of bulk shift is called a DBS because both the advancing and receding bulk menisci are on the concave side of the $\operatorname{arcs} \bar{\Gamma}_{\mathrm{o}}$ and $\Gamma_{\mathrm{o}}$, respectively. No bulk shift results for cases where $\Phi_{c}<0$ with $D_{1}=D_{2}$ for which $\Phi_{e}=\Phi_{g}=\Phi_{c} / 2$. However, the bulk shift immediately occurs when an infinitesimal displacement is applied to the vane for which $D_{1}<D_{2}$ and $\Phi_{e}<\Phi_{g}<0$ holds. Consistent with observations from both the experiments and numerical computations, it is again speculated that the bulk shift according to the C-singular solution in VPRe will take place because $\Phi_{e}$ is more negative than $\Phi_{g}$. Thus, the DBS occurs for infinitesimal lateral displacement of the vane.

For cases where $\Phi_{c}>0, \Phi_{e}=\Phi_{g}=\Phi_{c} / 2>0$ when $D_{1}=D_{2}$ and both $\Phi_{e}$ and $\Phi_{g}$ will not change sign for an infinitesimal lateral displacement of the vane. For cases where $D_{1}<D_{2}$, Eq. (3.7) shows that for any gap size $w$ that makes $\Phi_{c}>0$ there is a critical value for $D_{1}$ that may be expressed as

$$
D_{1 \mathrm{cr}}=\frac{(\pi-4) L_{1}^{2} C-4 L_{1}\left(L_{2}-2 w\right)(C-2 w)}{4 L_{1}-2 L_{2}+4 w},
$$

where $L_{1} \equiv D_{1}+D_{2}$ and $L_{2} \equiv D_{1}+D_{2}+2 C$ are constant. $\Phi_{e} \leq 0$ when $D_{1} \leq D_{1 \text { cr }}$ and the global $\mathrm{C}$-singular solution is produced by superposing the C-singular solutions of VPRa onto VPRe. As a result, the advancing bulk meniscus is connected to the receding bulk meniscus as shown in Fig. 12(b) with a quasi-steady numerically computed surface shown in Fig. 13(c). This type of bulk

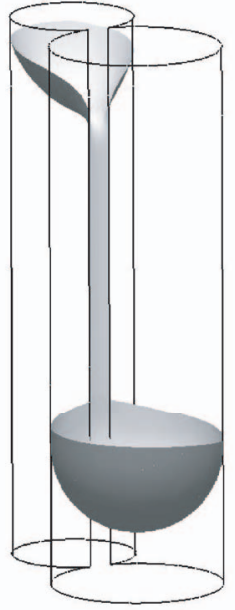

(a) CBS

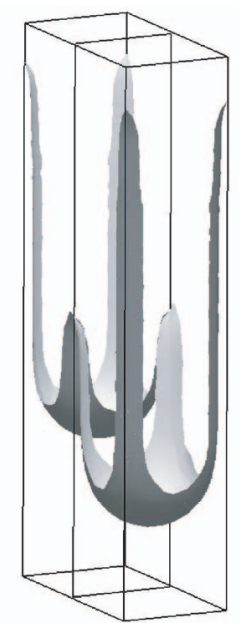

(b) DBS

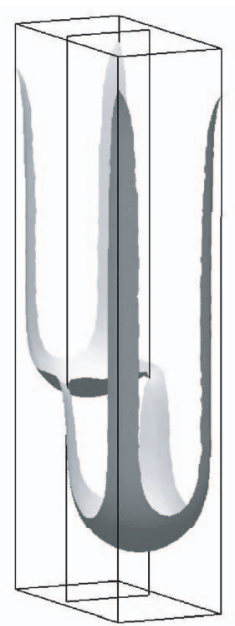

(c) CBS

FIG. 13. (a)-(c) Numerically computed quasi-steady surfaces in the asymmetric conjoined circular cylinders and the vanepartitioned rectangles. Note that the gap size in (b) is not zero. 
shift is called a CBS in which the advancing and receding bulk menisci share common arcs $\Gamma_{\mathrm{o}}$. To summarize, the CBS occurs only for finite lateral displacements of the vane. The surfaces in Fig. 13 are computed numerically using the open source SE-FIT ${ }^{23}$ software which adapts Brakke's Surface Evolver $^{24}$ algorithm. The containers of this paper are included in the SE-FIT pre-built geometries catalog.

Due to the difference between the DBS and CBS, different formulas must be used to evaluate the mean curvature of the advancing bulk meniscus. In what follows, the formulas for evaluating the mean curvature of the receding bulk meniscus is provided for convenience. For the DBS, the relevant terms of the receding meniscus are

$$
\Omega_{\mathrm{o}}=\Omega_{\mathrm{r}}-(4-\pi) R_{\mathrm{o}}^{2},
$$

with $\Omega_{\mathrm{r}} \equiv D_{2} C$ and $R_{\mathrm{o}} \equiv 1 / 2 H_{\mathrm{o}}$,

$$
\Sigma_{\mathrm{o}}=\Sigma_{\mathrm{r}}-8 R_{\mathrm{o}}
$$

with $\Sigma_{\mathrm{r}} \equiv 2\left(D_{2}+C\right)$, and

$$
\Gamma_{\mathrm{o}}=2 \pi R_{\mathrm{o}} .
$$

Substituting $\Omega_{\mathrm{o}}, \Sigma_{\mathrm{o}}$, and $\Gamma_{\mathrm{o}}$, Eq. (2.8) may be solved to obtain an expression for the mean curvature

$$
H_{\mathrm{o}}=\frac{4-\pi}{\Sigma_{\mathrm{r}}-\sqrt{\Sigma_{\mathrm{r}}^{2}-4(4-\pi) \Omega_{\mathrm{r}}}} .
$$

The first tier advancing and receding bulk menisci are separated by the second tier rivulet-like gap flows. As a result, the advancing bulk meniscus has similar characteristics as the receding bulk meniscus as it is on the concave side of the arcs that represent the $\mathrm{C}$-singular solutions in the corner and gap regions. The corresponding terms of the advancing bulk meniscus may be written as

$$
\bar{\Omega}_{\mathrm{o}}=\Omega_{\mathrm{ad}}-(4-\pi) \bar{R}_{\mathrm{o}}^{2},
$$

with $\Omega_{\mathrm{ad}} \equiv D_{1} \times C$ and $\bar{R}_{\mathrm{o}} \equiv 1 /\left(2 \bar{H}_{\mathrm{o}}\right)$,

$$
\bar{\Sigma}_{\mathrm{o}}=\Sigma_{\mathrm{ad}}-8 \bar{R}_{\mathrm{o}}
$$

with $\Sigma_{\text {ad }} \equiv 2\left(D_{1}+C\right)$,

$$
\bar{\Gamma}_{\mathrm{o}}=2 \pi \bar{R}_{\mathrm{o}}
$$

and for the DBS,

$$
\Gamma_{\mathrm{o}}=0
$$

Substituting $\bar{\Omega}_{\mathrm{o}}, \bar{\Sigma}_{\mathrm{o}}, \bar{\Gamma}_{\mathrm{o}}$, and $\Gamma_{\mathrm{o}}$, Eq. (2.12) may be solved to obtain a similar form for the mean curvature

$$
\bar{H}_{\mathrm{o}}=\frac{4-\pi}{\Sigma_{\mathrm{ad}}-\sqrt{\Sigma_{\mathrm{ad}}^{2}-4(4-\pi) \Omega_{\mathrm{ad}}}} .
$$

For the CBS, since the C-singular solutions in the two left corners of the rectangle are superposed onto the bulk shift $\mathrm{C}$-singular solution, the boundary of the advancing bulk meniscus comprises two circular $\operatorname{arcs} \bar{\Gamma}_{\mathrm{o}}$ in the corners whose mean curvature $\bar{H}_{\mathrm{o}}$ is to be determined. The relevant terms of the advancing bulk meniscus are written as

$$
\bar{\Omega}_{\mathrm{o}}=\Omega_{\mathrm{ac}}+\left(2-\frac{\pi}{2}\right) R_{\mathrm{o}}^{2}-\left(2-\frac{\pi}{2}\right) \bar{R}_{\mathrm{o}}^{2},
$$

with $\Omega_{\mathrm{ac}} \equiv D_{1} C$,

$$
\bar{\Sigma}_{\mathrm{o}}=\Sigma_{\mathrm{ac}}+4 R_{\mathrm{o}}-4 \bar{R}_{\mathrm{o}}
$$

with $\Sigma_{\mathrm{ac}} \equiv 2\left(D_{1}+C-2 w\right)$,

$$
\bar{\Gamma}_{\mathrm{o}}=\pi \bar{R}_{\mathrm{o}},
$$




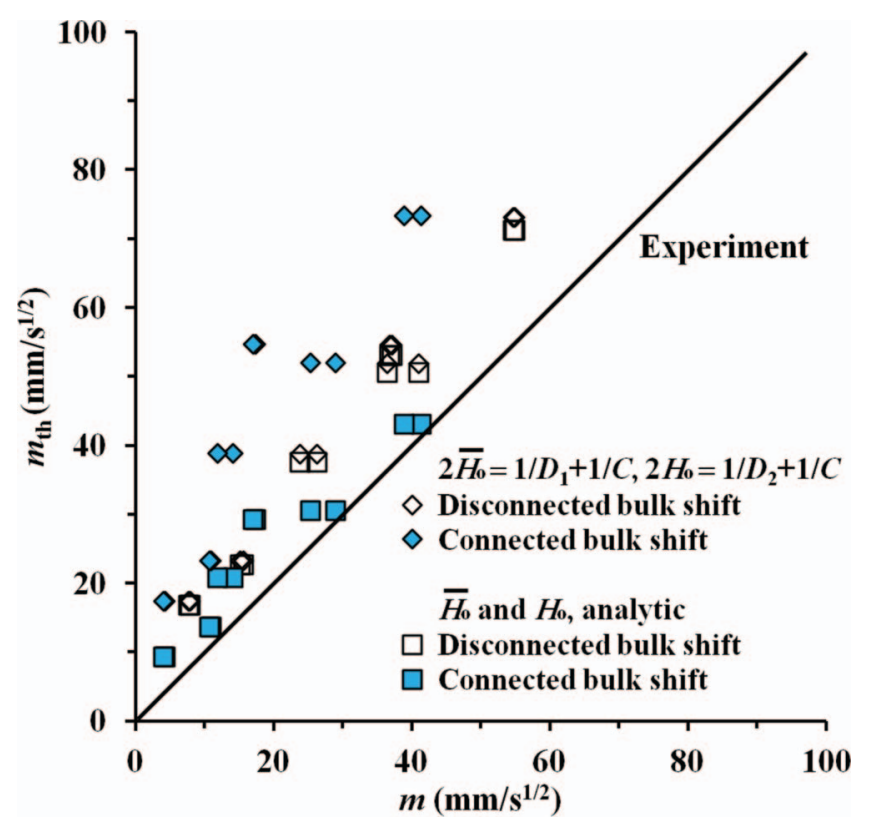

FIG. 14. A comparison of the coefficient $m$ (Eq. (3.4)), between experiment $(m)$ and calculations $\left(m_{\text {th }}\right)$ for vane-partitioned rectangles.

and

$$
\Gamma_{\mathrm{o}}=\pi R_{\mathrm{o}}
$$

Substituting $\bar{\Omega}_{\mathrm{o}}, \bar{\Sigma}_{\mathrm{o}}, \bar{\Gamma}_{\mathrm{o}}$, and $\Gamma_{\mathrm{o}}$, Eq. (2.12) may be solved to obtain an expression for the mean curvature

$$
\bar{H}_{\mathrm{o}}=\frac{2-\pi / 2}{\Sigma_{\mathrm{ac}}+(4-\pi) R_{\mathrm{o}}-\sqrt{\left(\Sigma_{\mathrm{ac}}+(4-\pi) R_{\mathrm{o}}\right)^{2}-(4-\pi)\left(2 \Omega_{\mathrm{ac}}+(4-\pi) R_{\mathrm{o}}^{2}\right)}} .
$$

In the study of $\operatorname{Tavan}^{19}$ the mean curvatures of the menisci are approximated with the dimensions of the rectangles on the two sides of the vane. Similar to the asymmetric conjoined circular cylinders, the mean curvatures of the menisci calculated with the formulations derived above are used to calculate $m$ in Eq. (3.4), which is then compared to the experimental results of Tavan in Fig. 14. On average, it appears that using the formulations of this study to calculate the mean curvatures, the flow model overpredicts the experimental results by $\approx 52 \%$ whereas using the dimensions of the rectangles the flow model overpredicts by $\approx 114 \%$. It is of interest to note that for compound capillary flows with a CBS the improvement is significant, whereas for compound flows with a DBS the improvement is slight as in those cases the present analytic mean curvature predictions are more in line with the approximations of Tavan.

The remaining discrepancies between the measurements and our predictions are still significant, but a concerted effort to resolve them is not attempted here. However, it is certain that the experiments never fully satisfied the conditions required to assure "quasi-steady" flow. For example, using the fluid properties and test cell dimensions of Tavan, ${ }^{19}$ it can be learned that inertial forces are significant from as low as $10 \%$ to as high as $50 \%$ of the $2.2 \mathrm{~s}$ drop tower test duration. Dynamic non-uniform contact angle effects are ignored in the analysis, which when estimated for the experiments using Hoffman's correlation ${ }^{25}$ establishes that $\gamma \neq 0$, but rather $20^{\circ} \lesssim \gamma_{\text {dyn }} \lesssim 30^{\circ}$. These elevated effective contact angles reduce the interface curvature and assure an overprediction by the analysis which assumes $\gamma_{\text {dyn }}=\gamma=0$. Dynamic models for capillary rise usually overpredict experimental results due to idealized assumptions, e.g., interior corner flows are over-predicted by typically $6 \%-7 \%$. $^{26}$ In addition, the unaccounted and non-uniform dynamic contact angle, the finite thickness of the vane, and other known uncertainties of test cell fabrication ${ }^{19}$ offer an additional cause of suspicion 
as to the accuracy, or even validity, of the experimental comparisons. Further experiments with finer control of conditions targeting comparisons with the present analytical method are recommended.

\section{SUMMARY}

It is shown that the mean curvature of advancing bulk menisci under bulk shift flows can be identified analytically in an asymptotic quasi-steady sense. This study serves as a foundation for a zeroth order dynamic analysis of such flows as in the case of the CFE Vane-Gap geometries. A preliminary Concus-Finn analysis of the CFE Vane-Gap geometry can be found in Chen et al. ${ }^{27}$ and a comprehensive study of the dynamics of the flow will be reported separately.

It is realized that there are two types of bulk shift flows. The disconnected bulk shift is one in which the advancing bulk meniscus is separated from the receding bulk meniscus by other advancing interfaces resulted from capillary flows at higher hierarchical levels. The connected bulk shift is one in which the advancing bulk meniscus is directly connected to the receding bulk meniscus. For equilibrium contact angle conditions, it is found that infinitesimal asymmetries below fabrication tolerance can cause disconnected bulk shifts to occur, whereas connected bulk shifts require a finite displacement. The magnitude of such displacements may be computed. These characteristic can be exploited for manipulating fluids.

For the geometries examined herein, it is relatively easy to estimate the mean curvature of the bulk menisci using the characteristic lengths of the container section. However, the new analytic method proves valuable when such estimations become problematic as a result of increased geometric complexity. Even when characteristic lengths are obvious, the method is especially useful to predict connected bulk shift capillary flows.

\section{ACKNOWLEDGMENTS}

Support for this work is primarily provided by the National Aeronautics and Space Administration (NASA) under cooperative agreement NNX09AP66A managed by NASA's Glenn Research Center (GRC). Incremental contributions under previous funding sources such as NASA NNC05AA29A and National Science Foundation (NSF) CTS-0521890 are also acknowledged. The authors thank Y. Reichel for providing helpful comments.

${ }^{1}$ M. M. Weislogel and S. Lichter, "Capillary flow in an interior corner," J. Fluid Mech. 373, 349-378 (1998).

${ }^{2}$ M. M. Weislogel, "Capillary flow in containers of polygonal section," AIAA J. 39, 2320-2326 (2001).

${ }^{3}$ T. Cubard and C.-M. Ho, "Transport of bubbles in square microchannels," Phys. Fluids 16, 4575-4585 (2004).

${ }^{4}$ Y. Chen and S. H. Collicott, "Experimental study on the capillary flow in a vane-wall gap geometry," AIAA J. 43, 2395-2403 (2005).

${ }^{5}$ D. A. Bolleddula, Y. Chen, B. Semerjian, N. Tavan, and M. M. Weislogel, "Compound capillary flows in complex containers: drop tower test results," Microgravity Sci. Technol. 22, 475-485 (2010).

${ }^{6}$ M. M. Weislogel, R. Jenson, Y. Chen, S. H. Collicott, J. Klatte, and M. Dreyer, "The capillary flow experiments aboard the International Space Station: Status," Acta Astronaut. 65, 861-869 (2009).

${ }^{7}$ A. de Lazzer, D. Langbein, M. Dreyer, and H. J. Rath, "Mean curvature of liquid surfaces in cylindrical containers of arbitrary cross-section," Microgravity Sci. Technol. IX, 208-219 (1996).

${ }^{8}$ M. M. Weislogel, J. A. Baker, and R. M. Jenson, "Quasi-steady capillary-driven flows in slender containers with interior edges," J. Fluid Mech. 685, 271-305 (2011).

${ }^{9}$ R. Finn and R. W. Neel, "C-singular solutions of the capillary problem," J. Reine Angew. Math. 512, 1-25 (1999).

${ }^{10}$ M. M. Weislogel and S. H. Collicott, "Capillary rewetting of vaned containers: Spacecraft tank rewetting following thrust resettling," AIAA J. 42, 2551-2561 (2004).

${ }^{11}$ Y. Chen, M. M. Weislogel, and C. L. Nardin, "Capillary-driven flows along rounded interior corners," J. Fluid Mech. 566, 235-271 (2006).

${ }^{12}$ N. Ichikawa, K. Hosokawa, and R. Maeda, "Interface motion of a capillary-driven flow in rectangular microchannel," J. Colloid Interface Sci. 280, 155164 (2004).

${ }^{13}$ P. Concus and R. Finn, "On capillary free surfaces in the absence of gravity," Acta Math. 132, 177-198 (1974).

${ }^{14}$ R. Finn, "A subsidiary variational problem and existence criteria for capillary surfaces," J. Reine Angew. Math. 353, 196-214 (1984).

${ }^{15}$ R. Finn, Equilibrium Capillary Surfaces, A Series of Comprehensive Studies in Mathematics Vol. 284 (Springer-Verlag, New York, 1986), Chaps. 6 and 7.

${ }^{16}$ P. Concus and R. Finn, "Continuous and discontinuous disappearance of capillary surfaces," in Variational Methods for Free Surface Interfaces, edited by P. Concus and R. Finn (Springer-Verlag, Berlin, 1987), pp. 197-204. 
${ }^{17}$ J. Lekan, D. Gotti, A. J. Jenkins, J. C. Owens, and M. R. Johnston, "Users guide for the 2.2 second drop tower of the NASA Lewis Research Center," Technical Memorandum No. 107090 (National Aeronautics and Space Administration, 1996).

${ }^{18}$ M. M. Weislogel, "Steady spontaneous capillary flow in partially coated tubes," AIChE J. 43, 645-654 (1997).

${ }^{19} \mathrm{~N}$. Tavan, "Critical geometric wetting phenomena: study of capillary driven flow in the CFE Vane-Gap experiment aboard the International Space Station," Master's thesis (Portland State University, Portland, OR, 2009).

${ }^{20}$ P. Concus and R. Finn, "On the behavior of a capillary surface in a wedge," Proc. Natl. Acad. Sci. U.S.A. 63, 292-299 (1969).

${ }^{21}$ P. Concus and R. Finn, “Capillary wedges revisited,” SIAM J. Math. Anal. 27, 56-69 (1996).

${ }^{22}$ Y. Chen and S. H. Collicott, "Investigation of the symmetric wetting behavior of vane-wall gaps in propellant tanks," AIAA J. 42, 305-314 (2004).

${ }^{23}$ Y. Chen, B. Schaeffer, M. M. Weislogel, and G. Zimmerli, "Introducing SE-FIT: Surface Evolver - Fluid Interface Tool for studying capillary surfaces," AIAA Paper 2011-1319, 2011.

${ }^{24}$ K. A. Brakke, "The surface evolver," Exp. Math. 1, 141-165 (1992).

${ }^{25}$ R. Hoffman, "A study of the advancing interface. I. Interface shape in liquid-gas system," J. Colloid Interface Sci. 50, 228-241 (1975).

${ }^{26}$ M. M. Weislogel, "Capillary flow in an interior corner,” Ph.D. dissertation (Northwestern University, 1996).

${ }^{27}$ Y. Chen, R. M. Jenson, M. M. Weislogel, and S. Collicott, "Capillary wetting analysis of the CFE Vane-Gap geometry," AIAA Paper 2008-817, 2008. 
Physics of Fluids is copyrighted by the American Institute of Physics (AIP). Redistribution of journal material is subject to the AIP online journal license and/or AIP copyright. For more information, see http://ojps.aip.org/phf/phfcr.jsp 